\title{
Low-cost and effective fabrication of biocompatible nanofibers from
}

\section{silk and cellulose-rich materials}

Susana Guzman-Puyol ${ }^{a, *}$, José A. Heredia-Guerrero ${ }^{a}$, Luca Ceseracciu ${ }^{a}$, Hadi Hajiali ${ }^{a, b}$, Claudio

Canale $^{c}$, Alice Scarpellini ${ }^{d}$, Roberto Cingolani ${ }^{a, c, d}$, Ilker S. Bayer $^{a}$, Athanassia Athanassiou $^{a, *}$, Elisa Mele $^{a, \dot{T}, *}$

${ }^{a}$ Smart Materials, Istituto Italiano di Tecnologia, Via Morego, 30, Genova, 16163, Italy

${ }^{b}$ DIBRIS, University of Genoa, Via Opera Pia, 13, Genova, 16145, Italy

${ }^{c}$ Nanophysics, Istituto Italiano di Tecnologia, Via Morego, 30, Genova, 16163, Italy

${ }^{d}$ Nanochemistry, Istituto Italiano di Tecnologia, Via Morego, 30, Genova, 16163, Italy

E-mail addresses: susana.guzman@iit.it, athanassia.athanassiou@iit.it, e.mele2@lboro.ac.uk

${ }^{\dagger}$ Present address: Department of Materials, Loughborough University, Loughborough, Leicestershire, LE11 3TU, UK

The data contained in this supplementary data are:

1. Deconvolution of amide I absorption for fibroin, MCC:fibroin, and parsley:fibroin nanofibers.

2. SEM image of parsley:fibroin. 
Figure S1. Deconvolution of amide I absorption for fibroin, MCC:fibroin, and parsley:fibroin nanofibers. Black line represents the real absorption, while color lines represent the different contributions. They are marked as random coil (R), beta-sheets (B), alpha-helices (A), turns (T), and side chains (SC).

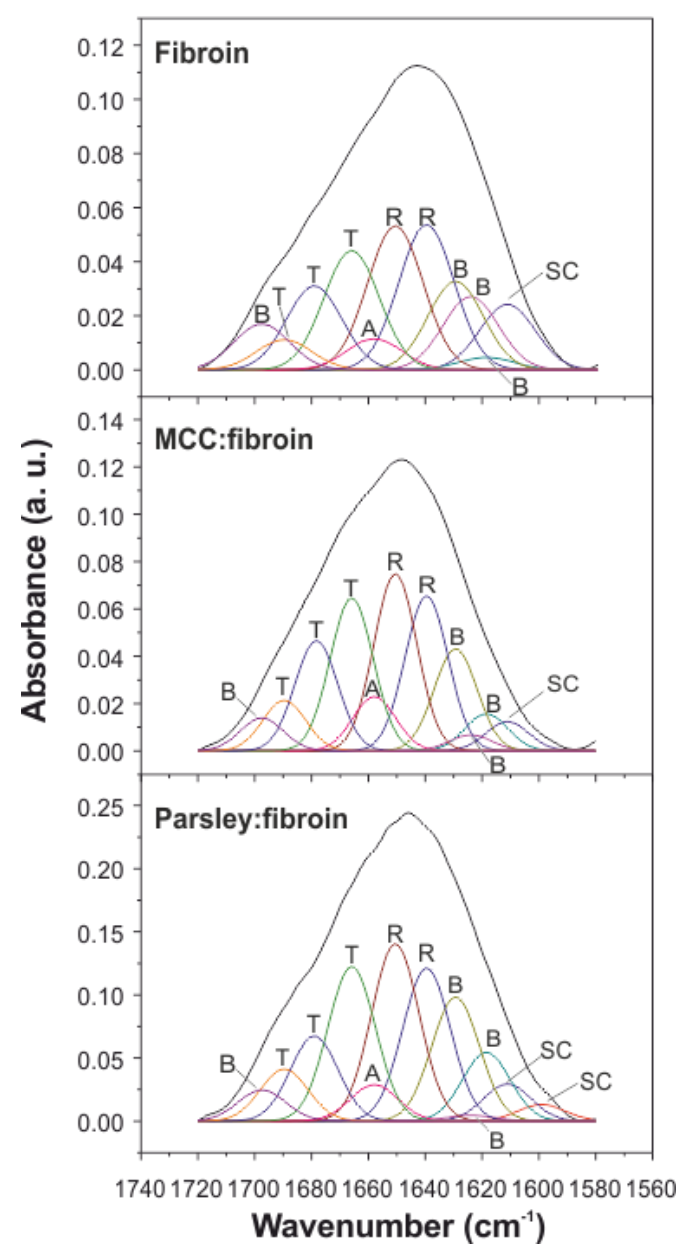


Figure S2. SEM image of parsley:fibroin. Detail of the electrospun parsley:fibroin nanofibers showing the crimped waveform organization of the fibers.

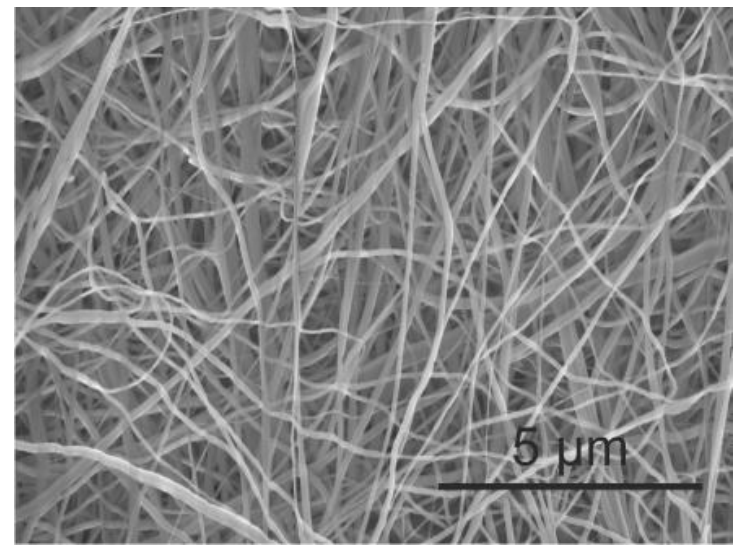

